\title{
Os reflexos da COVID-19 na farmacoterapia em pacientes portadores de doenças
}

\section{crônicas}

The effects of COVID-19 on pharmacotherapy in patients with chronic diseases

El alcance del COVID-19 sobre la farmacoterapia en pacientes con enfermedades crónicas

Recebido: 18/11/2021 | Revisado: 26/11/2021 | Aceito: 01/12/2021 | Publicado: 11/12/2021

Danielle Portugal Vieira

ORCID: https://orcid.org/0000-0002-9511-534X

Faculdade Independente do Nordeste, Brasil E-mail: dany_portugal@outlook.com.br

Nicolly Luz Pita

ORCID: https://orcid.org/0000-0002-4944-832X Faculdade Independente do Nordeste, Brasil

E-mail: nicollyluz@gmail.com

Tayanne Andrade dos Santos

ORCID: https://orcid.org/0000-0002-7977-6308

Faculdade Independente do Nordeste, Brasil

E-mail: tayanne.as@hotmail.com

Daianne Arruda Luz

ORCID: https://orcid.org/0000-0002-2734-2612

Faculdade Independente do Nordeste, Brasil

E-mail: daianne.arruda13@gmail.com

\begin{abstract}
Resumo
A pandemia da COVID-19, ocasionada por uma nova cepa de coronavírus, gerou grandes desafios por todo o mundo. Essa doença tem o poder de desenvolver maiores complicações, em relação a outras doenças, e uma maior ocorrência de internações em pacientes com hipertensão arterial sistêmica e diabetes mellitus. Este estudo teve como objetivo avaliar os reflexos da COVID-19 na farmacoterapia em pacientes portadores de doenças crônicas. Tratou-se de uma pesquisa de campo, descritiva, exploratória e de abordagem qualitativa e quantitativa, realizada no Ambulatório de Saúde da FAINOR, em setembro de 2021. Compuseram a amostra deste estudo 20 pacientes, tendo como critério de inclusão portadores de doenças crônicas, exclusivamente, hipertensão e diabetes que tiveram a doença COVID-19. E como critério de exclusão pacientes que não tiveram a doença. Os dados foram coletados através de um questionário aplicado em formato eletrônico. Para a avaliação e interpretação dos dados obtidos foi recorrido ao método do discurso do sujeito coletivo. Dos pacientes diagnosticados, cerca de 50\% apresentavam diabetes, $20 \%$ apresentavam hipertensão, 30\% diabetes e hipertensão associadas e 30\% apresentavam obesidade/sobrepeso. Cerca de $70 \%$ dessas pessoas desenvolveram sequelas pelo coronavírus, como desidratação, dores no corpo, tosse seca, esquecimento e Insuficiência Renal Aguda. Cerca de 40\% possuíam interações medicamentosas graves em seus tratamentos crônicos, concomitante com o tratamento do SARS-CoV-2. Concluiu-se que, a pandemia do COVID-19 não gerou mudanças perceptíveis na farmacoterapia dos participantes hipertensos e diabéticos. No entanto, o vírus refletiu negativamente no estado geral de saúde dos pacientes fazendo com que muitos ficassem com sequelas.
\end{abstract}

Palavras-chave: COVID-19; Hipertensão; Diabetes; Farmacoterapia.

\begin{abstract}
The COVID-19 pandemic, caused by a new strain of coronavirus, has created great challenges throughout the world. This disease has the power to develop complications, higher morbidity and mortality when compared to other diseases, and a higher prevalence of hospitalizations in patients with Systemic Arterial Hypertension (SAH) and Diabetes Mellitus (DM). This study aimed to evaluate the effects of COVID-19 on pharmacotherapy in patients with chronic diseases. This is a descriptive, exploratory research with a qualitative and quantitative approach, carried out at FAINOR's Health Clinic. The sample for this study consisted of 20 patients, with the inclusion criteria being patients with chronic diseases, exclusively SAH or DM who had COVID-19 disease. As exclusion criteria, patients who did not have the disease. The data were collected through a questionnaire applied in an electronic format. Of the patients diagnosed, about 50\% had isolated DM, 20\% had isolated hypertension, 30\% had associated DM and hypertension, and $30 \%$ were obese/overweight. About $70 \%$ of these people developed sequelae from the coronavirus, such as dehydration, body aches, dry cough, forgetfulness, and Acute Renal Failure. Approximately 40\% of these patients had serious drug interactions in their treatments for SAH and DM, as well as concomitant SARS-CoV-2 treatment. It was concluded from the data collected in the survey that for SAH and DM the questionnaire elicited responses that indicated that the pandemic COVID-19 did not generate noticeable changes in the pharmacotherapy of the
\end{abstract}


participants. However, the virus reflected negatively on the general health condition of the patients, causing sequelae in many of them.

Keywords: COVID-19; Hypertension; Diabetes; Pharmacotherapy.

\begin{abstract}
Resumen
La pandemia del COVID-19, generada por una nueva cepa de coronavirus, planteó grandes retos alrededordel mundo. Comparándolaconotrasenfermedades, estapuededesarrollarcomplicaciones, mayormorbilidad y mortalidad y una mayorprevalencia de hospitalizacionesen pacientes conHipertensión Arterial Sistémica y Diabetes Mellitus. El objetivo de este estudiofueevaluarel alcance del COVID-19 sobre la farmacoterapia en pacientes conenfermedades crónicas. Esta es una investigacióndescriptiva, exploratoriacon enfoque cualitativo y cuantitativo que se llevó a cabo enelAmbulatorio de Salud de la FAINOR. La muestra de este estudiolacompusieron 20 pacientes, estableciéndose como criterio de inclusión a aquellosconenfermedades crónicas, exclusivamente HAS o DM que tuvieronlaenfermedad COVID-19. Y como criterio de exclusión a los pacientes que no tuvieronlaenfermedad. Los datos se recolectaron a través de una encuesta aplicada electrónicamente. Alrededordel 50\% de los pacientes diagnosticados teníasólo DM, el 20\% sóloHipertensión, el 30\% DM y HAS asociadas y el 30\% del total teníaobesidad/sobrepeso. Alrededordel $70 \%$ de esas personas después de infectarseconcoronavirusdesarrollaronsecuelas como deshidratación, dolorescorporales, tos seca, olvido e Insuficiencia Renal Aguda. Alrededordel 40\% tuvointeracciones medicamentosas graves ensutratamiento para HAS y DM, como también, concomitantes coneltratamientodel SARS-CoV-2. Se concluye que losdatosrecogidos a través de laencuestaindican que la pandemia COVID-19 no generó grandes cambios para HAS y DM enla farmacoterapia de los participantes. Sin embargo, se percibe que elvirusimpactó negativamente lasalud general de los pacientes al dejarlessecuelas.
\end{abstract}

Palabras clave: COVID-19; Hipertensión; Diabetes; Farmacoterapia.

\title{
1. Introdução
}

A pandemia da COVID-19, iniciada em dezembro de 2019, causada por uma nova cepa de coronavírus, ocasionou muitos danos e grandes desafios para vários países do mundo. Associada especialmente a sintomas respiratórios, a doença denominada SARS-CoV-2 (Síndrome Respiratória Aguda Grave Coronavírus 2), tem se mostrado altamente contagiosa e letal devido a sua capacidade de transmissão rápida e sintomas atípicos ou até mesmo infecção assintomática (Guan; 2020; Lana et al., 2020).

A Organização Mundial de Saúde (OMS) desde o início da pandemia declarou a doença como uma emergência de saúde pública, alertando e aconselhando o mundo a implementar medidas para detecção precoce, promoção do distanciamento social assim como, respeitar a quarentena, enfatizando a necessidade de agilizar as pesquisas para o desenvolvimento de vacinas. O vírus se propagou por todos os países deixando consequências para as populações de risco, como hipertensos e diabéticos (Who, 2020; Zhu, 2020).

A COVID-19 tem o potencial de desenvolver complicações como a síndrome do desconforto respiratório agudo (ARDS), falência de múltiplos órgãos e choque. Vários fatores estão correlacionados com a maior mortalidade, maior prevalência de internações e complicações do coronavírus, a exemplo das doenças crônicas como a hipertensão arterial sistêmica (HAS) e diabetes mellitus (DM) (Lopez, 2020; Zheng, 2020).

A prevalência e taxa de mortalidade em pacientes diabéticos na Unidade de terapia intensiva (UTI) em comparação com outras doenças menos graves foram bem mais altas. Segundo o boletim epidemiológico de 21 a 27 de fevereiro de 2021 , disponibilizado pelo Ministério da Saúde, foram notificados 30.305 óbitos por COVID-19. Cerca de 9.904 possuíam pelo menos uma comorbidade, sendo que os cardiopatas (14.865 óbitos) e diabéticos (10.583 óbitos) foram as condições mais frequentes. Em um dos estudos usando a escala de avaliação de qualidade Newcastle-Ottawa, demonstrou que entre 1.382 pacientes (idade média de 51,5 anos, 798 homens), o DM foi a segunda comorbidade mais frequente. (Onder, 2020; Brasil, 2021).

Dentre as comorbidades mais comuns associadas ao COVID-19 está a HAS. Estudos recentes apontam que essa relação é devido à fisiopatologia das duas doenças envolvendo o sistema renina-angiotensina-aldosterona, que seria o principal 
receptor do coronavírus e fundamental na manutenção da homeostase da pressão arterial. Vale ressaltar que além da fisiopatologia da HAS e DM, existem condições de saúde apresentadas por esses pacientes que se tornam fatores de risco importantes como o sedentarismo, obesidade e a maioria com idade avançada (Abajo, 2020; Flacco, 2020).

Neste contexto, é necessário conhecer como o vírus se comporta e avaliar quais são os fatores de risco que favorecem o desenvolvimento e a progressão do COVID-19. Com isso, é possível entender os reflexos que ele pode causar a população e na farmacoterapia concomitante de ambas comorbidades, sejam esses o uso irracional de medicamentos, interações medicamentosas, reações adversas, entre outros (Aronson, 2020; Maddaloni, 2020).

Diante disso, e reconhecendo a importância da compreensão das diversas repercussões da infecção pelo SARS-CoV2, o objetivo deste estudo é avaliar os reflexos da COVID 19 na farmacoterapia em pacientes portadores de doenças crônicas: Hipertensão Arterial Sistêmica e Diabetes Mellitus.

\section{Metodologia}

Este estudo caracteriza-se como uma pesquisa de campo com finalidade descritiva e exploratória, apresentando uma abordagem qualitativa envolvendo todas as questões abertas de definição e quantitativa representando os dados numéricos (idade, sexo, quantidade de pacientes acometidos), na qual houve coleta de informações para serem descritas e analisadas, atentando para um maior número de elementos, a fim de uma compreensão mais apurada do problema estudado.

$\mathrm{Na}$ execução deste propósito, o estudo trabalhou com o procedimento técnico levantamento, que representa, segundo Gil (2008), um procedimento que envolve a interrogação direta das pessoas em busca de informações acerca do problema estudado.

Essa pesquisa foi realizada em uma Faculdade do interior da Bahia com os pacientes atendidos no Ambulatório de Saúde da instituição. Esse setor oferece gratuitamente serviços de atenção farmacêutica (aferição de pressão arterial, dosagem de glicemia capilar e plano de cuidado), descarte de medicamentos vencidos e integra a saúde da mulher, do homem, do idoso e da criança.

Para o cálculo da amostra foi realizada uma análise estatística com grau de confiança de 95\% e margem de erro de $5 \%$, tendo como população atendidos no ambulatório 20 pessoas, sendo assim, compuseram a amostra 20 pacientes. Esse estudo foi realizado com uma proposta e finalidade de controle dos pacientes atendidos no ambulatório. Obteve como critério de inclusão portadores de doenças crônicas, exclusivamente, HAS ou DM que tiveram a doença COVID-19. E como critério de exclusão pacientes que não tiveram a doença. Todos os participantes concordaram com os Termos de Consentimento Livre e Esclarecido, conforme a Resolução nº 466/2012 da Comissão Nacional de Saúde.

A coleta de dados se deu através de um questionário para uma análise mais objetiva e clara da situação atual da farmacoterapia dos pacientes selecionados. Foi aplicado em formato eletrônico, via e-mail, para acesso através de link gerado por meio de uma ferramenta gratuita oferecida pelo Google: o Google Forms. O questionário, dividido em duas partes, trouxe em sua primeira etapa os aspectos do perfil da população investigada (idade, gênero, escolaridade, estado civil, raça) e a segunda etapa dados da sua comorbidade (diagnóstico, estilo de vida, diferenças notadas após a infecção do vírus), farmacoterapia (prescrições, uso irracional de medicamentos, interações medicamentosas e adesão terapêutica) e a COVID-19 (diagnóstico, vacinação, sequelas).

Ao término da coleta de dados estes foram transcritos e tabulados utilizando-se uma planilha do programa Microsoft Office Excel®2019. A partir da análise das respostas do questionário efetuou-se uma coleta de dados documentais e bibliográficos, para fundamentar a pesquisa. Para a avaliação e interpretação dos dados coletados foi recorrido ao método do discurso do sujeito coletivo.

No discurso do sujeito coletivo, como procedimento, são feitas entrevistas individuais com questões abertas, 
resgatando o pensamento, podendo ser divulgado e preservando a sua característica qualitativa (Lefèvre, 2005; Lefrèvre, 2009).

A pesquisa foi submetida ao Comitê de Ética e Pesquisa de uma Faculdade do interior da Bahia e conduzida de acordo com a Resolução n 466/2012 da Comissão Nacional de Saúde, sendo realizada após aprovação sob o Parecer Consubstanciado $\mathrm{n}^{\mathrm{o}} 4.870 .181$.

\section{Resultados e Discussão}

A partir dos dados coletados no questionário obteve-se 20 respostas totais de pacientes que apresentavam DM e/ou HAS, diagnosticados com coronavírus. Desse modo, as respostas foram analisadas e discutidas ao longo do artigo, uma vez que esses participantes responderam satisfatoriamente à pesquisa.

Observou-se que $100 \%$ dos pacientes obtiveram duas doses disponibilizadas da vacina. Estudos realizados comprovaram os desafios que o SARS-CoV-2 apresentou para o desenvolvimento de vacinas, gerando uma corrida global para sua produção e tratamentos em tempo recorde. A vacinação é uma das conquistas humanas mais importantes, reduzindo com êxito a carga de doenças infecciosas por todo o mundo. Além disso, pode ser utilizada para controlar a morbimortalidade e reduzir a gravidade da doença para aqueles que foram diagnosticados (Greenwood, 2014; Milken Institute, 2020).

Todos os participantes da pesquisa possuíam diagnóstico para a doença. Nesse caso, para confirmar a infecção pelo coronavírus, 70\% dos participantes realizaram o exame do RT- PCR (Reação em cadeia da polimerase com transcrição reversa em tempo real) e $30 \%$ realizaram o teste rápido.

O RT-PCR é considerado padrão-ouro para identificação do RNA viral em pacientes na fase aguda da doença, inserido em diferentes protocolos de triagem em casos suspeitos. As amostras para essa análise são provenientes, principalmente, de swabs nasais e orofaríngeos, secreção traqueal e lavagem bronco-alveolar em pacientes críticos (Sbpc/Ml, 2020; Who, 2020). Esse exame fornece importantes informações nos estágios iniciais da infecção, quando a carga viral é mais baixa.

Ademais, permite diferenciar o SARS-CoV-2 de outros vírus semelhantes e por meio da deteç̧ão direta do patógeno é possível prevenir a transmissibilidade, apresentando alta sensibilidade e especificidade, respectivamente (Lima, 2020; Sbpc/Ml, 2020).

Neste estudo, a maioria dos pacientes que realizaram os testes para diagnóstico tiveram aparição de sintomas. Entre os mais relatados, estão a dor muscular e perda de paladar com $60 \%$ de manifestação; febre, tosse seca, dor de cabeça, perda de olfato, dor de garganta e coriza com $40 \%$ de manifestação; diarreia em $30 \%$ dos casos; falta de ar, náuseas e vômitos em $20 \%$ dos casos e, $10 \%$ não apresentaram nenhum sintoma. Os relatos das ocorrências entram em consonância com estudos que dizem que os sintomas da COVID- 19 não são específicos, em alguns pacientes há ausência desses indícios, no entanto, os casos sintomáticos podem se manifestar semelhantes ao da gripe, como dores de cabeça e no corpo, febre, coriza, tosse, fadiga etc. podendo evoluir para uma pneumonia e até morte, principalmente quando associados a comorbidades (Chaolin, 2020; Ferreira, 2020).

Dos pacientes diagnosticados, cerca de 50\% apresentaram DM isolada, 20\% apresentavam HAS isolada, 30\% DM e HAS associadas e 30\% apresentavam obesidade/sobrepeso. A idade dos participantes que responderam à pesquisa foi representada em escala, sendo assim, obteve-se os seguintes resultados: 20-30 anos (10\%); 30-40 anos (20\%); 40-50 anos (30\%); 50-60 anos (10\%); 60-70 anos (30\%). Dentre esses indivíduos 50\% eram do sexo feminino e 50\% do sexo masculino.

Em relação à pandemia, uma das maiores preocupações é a respeito da susceptibilidade de alguns grupos de riscos aos efeitos da COVID-19. Os pacientes que apresentam fatores de riscos como a DM e HAS, estão sujeitos a desenvolver a forma grave da doença, além de complicações cardiovasculares (Guo, 2020; Zhou, 2020). 
Entre os fatores de risco para a HAS e DM estão os não modificáveis como, idade, sexo, raça, histórico familiar, e os modificáveis como, sobrepeso ou obesidade, sedentarismo, ingestão de sódio e potássio, consumo de álcool, tabagismo, entre outros. A HAS não controlada e associada a fatores de risco metabólicos como, dislipidemia, obesidade abdominal, e DM, podem gerar sinais e sintomas perceptíveis decorrentes de possíveis complicações, por isso a necessidade de conhecer a patologia em questão (Arnett, 2019; Barroso, 2020).

A HAS é uma doença crônica não transmissível (DCNT), é uma condição clínica multifatorial caracterizada por níveis elevados e persistentes da pressão arterial (PA), ou seja, PA sistólica (PAS) maior ou igual a $140 \mathrm{mmHg}$ e/ou PA diastólica (PAD) maior ou igual a $90 \mathrm{mmHg}$, valor de referência para medições em consultório (Brasil, 2013; Précoma, 2019).

As doenças cardiovasculares (DCV) são consideradas atualmente como a principal causa de morbimortalidade, sendo a HAS constituída como uma das mais importantes. Em 2017, dados completos e revisados do Datasus mostraram a ocorrência de 1.312.663 óbitos no total, com um percentual de 27,3\% para as DCV. No ano de 2016, essa patologia foi responsável por cerca de 49640 óbitos, evoluindo para o quadro de 388 mortes por dia no ano de 2018 (Brasil; 2017; Brasil, 2019b).

Em relação a DM, se trata de um grupo de doenças metabólicas caracterizado por hiperglicemia persistente, associada a complicações, disfunções e insuficiência de vários órgãos, como olhos, rins, nervos, cérebro, coração e vasos sanguíneos. Essa doença é decorrente do déficit na produção de insulina ou na sua atividade, ou em ambos os mecanismos. Atinge dimensões epidêmicas, com estimativa de 415 milhões de portadores de DM mundialmente (Idf, 2015; Who, 2016).

Segundo avaliação da OMS, o número de portadores da doença em todo o mundo era de 177 milhões no ano 2000, com probabilidade de alcançar 350 milhões de pessoas em 2025. Os tipos de DM mais frequentes são o DM tipo 1, DM tipo 2 e DM gestacional. Outros tipos de DM ocasionais podem resultar de defeitos genéticos da função das células beta, defeitos na ação da insulina, efeito adverso de medicamentos, entre outros, gerando consequências principalmente para pessoas com idade avançada (Ada, 2017; Skyler, 2017).

Até o dia 03 de junho, ocorreram 35.126 óbitos de pessoas idosas no Brasil, correspondendo a 71\% do total de óbitos por COVID-19, segundo dados do SIVEP-Gripe (Sistema de Informação de Vigilância Epidemiológica da Gripe). Durante a pandemia foi notória a vulnerabilidade de idosos com comorbidades em relação ao coronavírus, pois foram os que mais se infectaram e sofreram com os impactos trazidos pela doença. Pessoas com idade avançada geralmente têm o sistema imunológico mais frágil, podendo ser por motivo natural pelo próprio processo de envelhecimento ou doenças crônicas e até mesmo pela quantidade de medicamentos utilizados (polifarmácia), associados a um modo de vida inadequado para o bemestar dos mesmos (Icict, 2021; Romero, 2021).

Segundo estudos de García-Álvarez (2020) e Bhutani (2020), o início da pandemia trouxe mudanças no estilo de vida das pessoas, como a diminuição da prática de atividade física, consumo de alimentos industrializados e bebidas alcóolicas. A preocupação com o vírus e a perda dos empregos, levaram os indivíduos a passarem mais tempo em suas casas devido o isolamento e distanciamento social, influenciando no aumento do sedentarismo e em um modo de vida inadequado para a preservação da saúde (Bhutani, 2020; García, 2020).

De acordo com os dados coletados nos pacientes do ambulatório, percebeu-se que $60 \%$ diziam não consumir bebidas alcóolicas, $20 \%$ consumiam socialmente, $10 \%$ ingeriam mais de duas vezes por semana e $10 \%$ não consumiam. No que se trata de atividade física durante a pandemia, cerca de $40 \%$ relataram não praticar nenhum tipo de exercício físico, $20 \%$ praticavam academia e musculação e 60\% caminhada, sendo que alguns deles começaram suas atividades após início do COVID-19.

Embora o fechamento dos locais das práticas de exercícios físicos, como academia, tenha contribuído para o sedentarismo, convém destacar que, como a maioria dos participantes, algumas pessoas mantiveram seus treinos ao ar livre priorizando os aspectos de segurança. Os benefícios dessa continuidade durante a pandemia incluem uma melhora na 
percepção do estresse, qualidade do sono, qualidade de vida, ansiedade e depressão, inclusive no tratamento daqueles infectados (Costa, 2020; Solon Júnior et al., 2020).

A infecção por SARS-CoV-2 dispõe de muitos detalhes a serem esclarecidos, incluindo o seu tratamento. Até então, não há informações concretas sobre a história natural e sobre a condução clínica da doença. No entanto, existem protocolos de condução terapêutica que difere os casos leves e graves (Mcintosh, 2020; Who, 2020).

Os casos leves, de forma geral, se baseiam na tentativa de suporte e conforto para o paciente, isolamento domiciliar e em alguns casos, supervisão até alta do isolamento (Who, 2020; Mcintosh, 2020). São indicadas nessa circunstância medidas não farmacológicas, como repouso, hidratação, alimentação adequada, garantir que o ambiente esteja ventilado, manter medidas de higiene como a lavagem das mãos e utilização de álcool em gel. Em alguns casos, são utilizados analgésicos e antitérmicos para diminuir os sintomas de dores e febre, respectivamente (Brasil, 2020; Who, 2020).

Por outro lado, os casos identificados como graves apresentando saturação de $\mathrm{SpO} 2>95 \%$ em ar ambiente, desconforto respiratório, hipotensão, comorbidades e piora nos sinais clínicos de base, são rapidamente encaminhados ao serviço de urgência e emergência (Huang, 2020; Ramanathan, 2020). Alguns fármacos utilizados nos casos graves de COVID19 envolvem as classes de Antibióticos, Corticosteroides, Antivirais, Inibidores da Enzima Conversora de Angiotensina (IECA) e Bloqueadores do Receptor de Angiotensina (BRA).

A seguir, na tabela 1 e 2 estão representados os principais medicamentos utilizados pelos participantes da pesquisa em relação a sua doença crônica e ao COVID-19, respectivamente. A maioria dos pacientes hipertensos e diabéticos necessitam de fármacos em adição às modificações do estilo de vida para alcançar a meta pressórica e glicêmica. Com ambas comorbidades é necessário observar a farmacoterapia utilizada para garantir o uso racional de medicamentos e entender quais são os reflexos que o coronavírus gera nos seus tratamentos (Task, 2017; Williams, 2018).

Tabela 1. Medicamentos para o tratamento de hipertensão e diabetes utilizados pelos pacientes do ambulatório.

\begin{tabular}{|c|c|c|c|}
\hline & Medicamento & Mecanismo de ação & Efeitos adversos \\
\hline \multirow{3}{*}{ 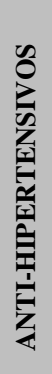 } & $\begin{array}{l}\text { Losartana } \\
\text { (BRA) }\end{array}$ & Bloqueia a ligação da angiotensina II. & Tosse, infecção do trato respiratório, tontura. \\
\hline & $\begin{array}{l}\text { Hidroclorotiazida } \\
\text { (Tiazídico) }\end{array}$ & Reabsorção de eletrólitos no túbulo distal. & $\begin{array}{l}\text { Efeitos gastrointestinais, cefaleia, } \\
\text { hipocalemia. }\end{array}$ \\
\hline & $\begin{array}{l}\text { Diovan HCT } \\
\text { (BRA) }\end{array}$ & Bloqueia a ligação da angiotensina II. & Hipercalemia, tontura, hipotensão, fadiga. \\
\hline \multirow{3}{*}{$\frac{0}{0}$} & $\begin{array}{l}\text { Glibenclamida } \\
\text { (Sulfoniluréia) }\end{array}$ & $\begin{array}{l}\text { Estimula a liberação de insulina das células beta } \\
\text { funcionantes das ilhotas do pâncreas. }\end{array}$ & Náuseas, azia, sensação de estômago cheio. \\
\hline & $\begin{array}{l}\text { Dapaglifozina } \\
\text { (SGLT2*) }\end{array}$ & $\begin{array}{l}\text { Com a inibição reduz a reabsorção de glicose e } \\
\text { diminui o limiar renal de glicose. }\end{array}$ & $\begin{array}{l}\text { Insuficiência renal, infecções micóticas, } \\
\text { poliúria, depleção de volume. }\end{array}$ \\
\hline & $\begin{array}{l}\text { Insulina Fiasp } \\
\text { (Ultrarrápida }\end{array}$ & $\begin{array}{l}\text { Reduzem a glicose no sangue, estimulando a } \\
\text { captação periférica de glicose. }\end{array}$ & $\begin{array}{c}\text { Hipoglicemia, reações no local da aplicação, } \\
\text { nasofaringite, diarreia. }\end{array}$ \\
\hline
\end{tabular}




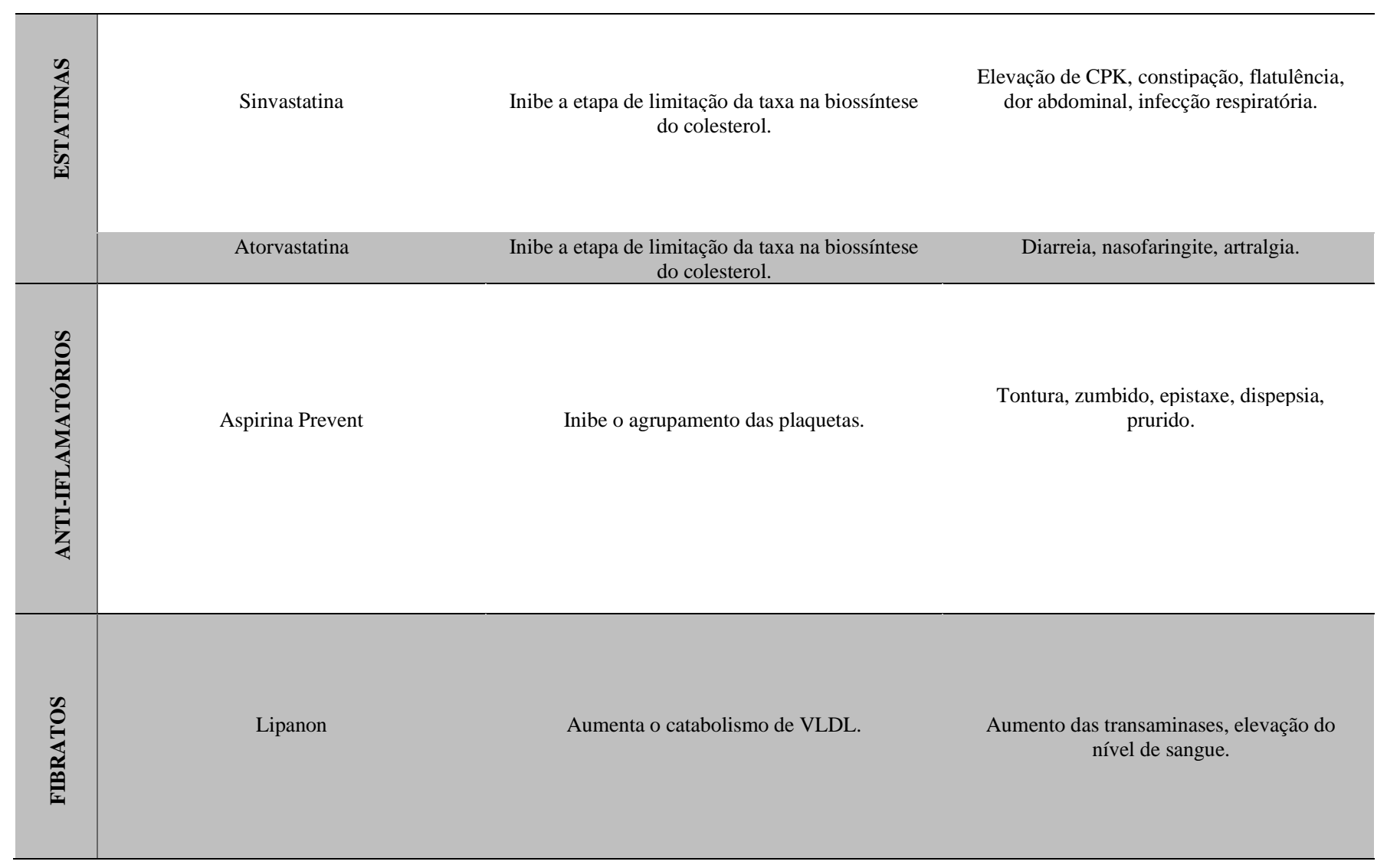

*SGLT: Inibidor seletivo do co-transportador de sódio-glicose tipo 2. Fonte: Autores.

Dentre os medicamentos descritos na Tabela 1, cerca de 100\% dos pacientes alegaram possuir prescrição médica dos fármacos para o tratamento da DM e HAS. Receituários de DM incluíam Metformina, Glibenclamida, Insulina Fiasp (Asparte) E Dapaglifozina, assim como as de HAS que incluíam Losartana, Hidroclorotiazida (HCTZ), Valsartana e HCTZ associados (Diovan). A monoterapia é utilizada como estratégia anti-hipertensiva inicial em pacientes com HAS estágio 1 com risco de doença cardiovascular (CV) baixo ou para indivíduos idosos e/ou frágeis, de modo a evitar eventos adversos. Enquanto, a combinação de fármacos para a maioria dos hipertensos é a estratégia terapêutica preferencial, independente do estágio da HAS e do risco CV associados (Póvoa, 2014; Wald, 2014).

As classes de anti-hipertensivos consideradas preferenciais para o controle da PA em monoterapia inicial são: diuréticos (DIU) tiazídicos ou similares, bloqueadores dos canais de cálcio (BCC), inibidores da enzima conversora de angiotensina (IECA), bloqueadores dos receptores da angiotensina II (BRA) e betabloqueadores (BB), porém, os betabloqueadores são considerados como fármaco inicial em situações específicas, e frequentemente em associação a outros fármacos (Barroso, 2020; Daskalopoulou, 2015). Entre os participantes, 20\% utilizavam losartana (BRA) isolada e 20\% associada a outras drogas.

Os pacientes que utilizavam apenas a losartana, após a COVID-19 relataram uma diferença nos controles pressóricos, alegando oscilação de seus valores. Alguns dos pacientes hipertensos e diabéticos avaliados declararam ficar com sequelas persistentes após infecção pelo coronavírus, como dores no corpo, tosse seca, insuficiência renal aguda (IRA) e esquecimento. A IRA é um fator considerável para a COVID-19, principalmente em pacientes com comorbidades, possuindo várias hipóteses de mecanismo como, por exemplo, a relação do vírus com os receptores dos rins ocasionando a lesão e a incidência de doenças como HAS e DM que já provocam problemas renais isoladas (Gabarre, 2020; Grasseli, 2020).

Uma das evidências contemporâneas foi a discussão de que o tratamento com anti-hipertensivos inibidores do sistema renina angiotensina aldosterona (IECAs) e os bloqueadores do receptor AT1 da angiotensina 2 (BRAs) poderiam influenciar na 
piora clínica da COVID-19 e controle da pressão arterial. Vários estudos fazem um enfoque para esta classe de medicamentos, insinuando que os IECAs ou BRAs podem crescer a expressão da ECA-2 no sistema cardiovascular e renal, aumentando o grau de infectividade e piora da infecção por SARS-CoV-2 em hipertensos (Gao, 2020; Vaduganathan, 2020).

Em controvérsia, outros autores observaram que estes fármacos podem ser benéficos na prevenção e tratamento da injúria pulmonar causado pela COVID 19. Eles atuam aumentando a expressão da ECA-2 agindo por mecanismos semelhantes reduzindo a pressão arterial. Duas hipóteses vêm sendo formuladas em relação ao efeito deletério vs protetor, sendo assim, enquanto não há estudos conclusivos sobre essa relação, o Conselho de Hipertensão da Sociedade Europeia de Cardiologia e as diretrizes recomendam não suspender os IECAs ou BRAs, pois são sabidamente cardioprotetores e renoprotetores, sendo essenciais ao tratamento (Lopes, 2020; Vaduganathan, 2020).

Acerca da DM, envolvendo apenas os antidiabéticos, relatados na tabela 1,30\% dos pacientes utilizavam metformina isolada, 20\% associada a outro antidiabético como glibenclamida e dapaglifozina, 10\% usavam apenas insulina Fiasp e entre os 100\% dos participantes, $20 \%$ utilizavam medicamentos para dislipidemia. Estudos demonstram que o SARS-CoV-2 penetra nas células humanas através da enzima conversora de angiotensina 2 (ECA2), encontradas também em células $\beta$ pancreáticas humanas, das quais o vírus pode agir alterando a sua função e prejudicando a secreção de insulina. Dessa forma, pode causar alterações no metabolismo da glicose complicando a fisiopatologia preexistente do diabetes ou ainda levar a novos mecanismos da doença. Portanto, é necessário um frequente monitoramento (Hollstein, 2020; Nejm, 2021).

Após análise das respostas dos pacientes do ambulatório, obteve-se relatos de sequelas marcantes dos diabéticos após COVID-19, entre eles, $10 \%$ desenvolveram a doença posterior a infecção pelo vírus e $10 \%$ desenvolveram desidratação, logo, sendo hospitalizados, $10 \%$ não obtiveram nenhuma sequela e os $70 \%$ foram em conjunto com a HAS.

O coronavírus causa danos direto às células das ilhotas pancreáticas, sendo possível, portanto, que este vírus possibilite alterações no metabolismo e na homeostasia da glicose e contribua com o início do DM em indivíduos susceptíveis, como nos pacientes do estudo, ou aumente a severidade das complicações associadas ao diabetes já explícito. Porém, ainda não há evidências concretas para sustentar a hipótese de um potencial efeito diabetogênico (redução dos efeitos da insulina) da COVID-19. Devido a altas concentrações de glicose no sangue, pacientes que vivem com a DM possuem grande risco de desidratação (Hussain, 2020; Rubino, 2020).

Com esses níveis de glicose alterados, o sistema renal demanda um maior trabalho para conseguir eliminá-la, levando consigo uma significante quantidade de água, que é importante para a imunidade como também para diminuir risco de agravamento pelo COVID-19 já que a desidratação também é um dos sintomas causados pela infecção do vírus. Uma das consequências encontradas na farmacoterapia do participante foi um dos efeitos adversos da Dapaglifozina que é a poliúria que, juntamente com o coronavírus resultaram na desidratação e sudorese exacerbada no paciente, optando pela retirada e substituição da dapaglifozina no internamento hospitalar (Davies, 2020; Deng, 2020).

Para controlar as manifestações do SARS-COV-2 é utilizada terapia farmacológica que envolvem as classes de Antibióticos, Corticosteroides, Antivirais, IECAs e Bloqueadores do Receptor de Angiotensina (BRA), descritos na Tabela 2 (Davies, 2020; Rubino, 2020). 
Tabela 2. Medicamentos para o tratamento da COVID-19 utilizados pelos pacientes do ambulatório.

\begin{tabular}{|c|c|c|c|}
\hline Medicamento & Classe & Mecanismo de Ação & Efeitos Adversos \\
\hline Dipirona & Analgésico e Antitérmico & $\begin{array}{l}\text { Inibição da ciclo-oxigenase (COX- } \\
1, \text { COX-2 ou ambas) }\end{array}$ & $\begin{array}{l}\text { Choque anafilático, dor no } \\
\text { estômago, arritmias cardíacas. }\end{array}$ \\
\hline $\begin{array}{l}\text { Tylenol Sinus (paracetamol } \\
\text { + pseudoefedrina) }\end{array}$ & $\begin{array}{c}\text { Analsésico + } \\
\text { descongestionante nasal }\end{array}$ & $\begin{array}{l}\text { Analgesia pela elevação do limiar } \\
\text { da dor e antipirese. } \\
\text { Agonita dos receptores } \alpha \text { e } \beta \\
\text { adrenérgicos, respectivamente }\end{array}$ & $\begin{array}{l}\text { Nervosismo, boca seca, náusea, } \\
\text { tontura, insônia. }\end{array}$ \\
\hline $\begin{array}{c}\text { Davena } \\
\text { (Pantoprazol) }\end{array}$ & Inibidor da bomba de prótons & $\begin{array}{c}\text { Inibidor da bomba de prótons } \\
\mathrm{H}+/ \mathrm{K}+\text { bloqueando secreção } \\
\text { gástrica }\end{array}$ & $\begin{array}{l}\text { Dor abdominal, cabeça, } \\
\text { diarreia, constipação. }\end{array}$ \\
\hline Hixizine (Hidroxizine) & Anti-histamínicos & $\begin{array}{c}\text { Inibe a liberação de histamina (H1) } \\
\text { e suas consequentes ações } \\
\text { sistêmicas }\end{array}$ & $\begin{array}{l}\text { Sedação, sonolência e secura na } \\
\text { boca. }\end{array}$ \\
\hline Prednisona & Corticoide & $\begin{array}{l}\text { Agonista do receptor de } \\
\text { glucocorticóide. }\end{array}$ & $\begin{array}{c}\text { Urticária, distúrbios } \\
\text { gastrointestinais, eletrolíticos. }\end{array}$ \\
\hline Ivermectina & Anti-Helmínticos & $\begin{array}{c}\text { Liga os canais de íons } \mathrm{Cl} \\
\text { dependentes de glutamato em } \\
\text { células nervosas e musculares de } \\
\text { invertebrados. }\end{array}$ & $\begin{array}{l}\text { Náusea, vômito, tontura, } \\
\text { astenia. }\end{array}$ \\
\hline Loratadina & Anti-histamínicos & $\begin{array}{c}\text { Antagonista seletiva do receptor } \mathrm{H} 1 \\
\text { de histamina periférica. }\end{array}$ & Sonolência, Dor de cabeça. \\
\hline Azitromicina & $\begin{array}{c}\text { Antibiótico } \\
\text { (Macrolídeos) }\end{array}$ & $\begin{array}{l}\text { Liga-se à subunidade ribossômica } \\
50 \mathrm{~S} \text { do ribossomo, inibindo a } \\
\text { síntese bacteriana. }\end{array}$ & Diarreia, náusea, dor abdominal \\
\hline Vitamina $\mathbf{C}$ & Vitamina (ácido ascórbico) & $\begin{array}{l}\text { Necessário para a formação de } \\
\text { colágeno e reparo de tecidos }\end{array}$ & $\begin{array}{l}\text { Diarreia e efeitos } \\
\text { gastrointestinais. }\end{array}$ \\
\hline $\begin{array}{c}\text { Koide D (Dexclorfeniramina } \\
\text { + Betametasona) }\end{array}$ & $\begin{array}{l}\text { Anti-histamínico e anti- } \\
\text { inflamatório }\end{array}$ & $\begin{array}{l}\text { Análogo do cortisol e Antagonista } \\
\text { receptor H1 (anti-histamíno), } \\
\text { respectivamente }\end{array}$ & $\begin{array}{c}\text { Sonolência, urticária, distúrbios } \\
\text { gastrointestinais, eletrolíticos. }\end{array}$ \\
\hline
\end{tabular}

Fonte: Autores.

A Tabela 2 descreve os medicamentos utilizados pelos pacientes durante a infecção, dentre os mais utilizados estão a dipirona, ivermectina, azitromicina, prednisona e paracetamol, no qual 60\% alegaram não sentir nenhum efeito após a utilização dos mesmos e $40 \%$ manifestaram sintomas como, empolação no corpo por excesso de dipirona, inchaço, queda de oxigenação e sudorese em excesso. Vale ressaltar que a utilização de alguns fármacos ainda é controversa, podendo gerar resultados positivos ou não (HUANG, 2020; Ramanathan, 2020).).

O tratamento do coronavírus é baseado principalmente no controle dos sintomas, sendo assim, a dipirona e o paracetamol são medicamentos recomendados pela OMS para esses casos, como analgésicos e antitérmicos. Porém, com a falta de informação, é utilizada sob automedicação de forma irracional podendo algumas vezes exceder as doses máximas diárias provocando efeitos adversos como náuseas, vômitos e até mesmo a empolação relatada por uma paciente (Fang, 2020; Who, 2020). 30\% dos participantes não possuíam prescrição médica para sua terapia contra a COVID-19. A farmacoterapia é ideal quando o paciente recebe todos os medicamentos que necessita, não utiliza nenhum medicamento impróprio, adere ao tratamento e quando é efetivo não produz um novo problema de saúde, nem agrava um já existente (Bouadma, 2020; Wang, 2020). 
Outro problema relatado foi a queda de oxigenação de um dos participantes após COVID-19, que fazia uso dos medicamentos ivermectina, azitromicina e prednisona, além da metformina e losartana para suas comorbidades. Esses pacientes de risco, que possuem doenças crônicas e acima de 60 anos devem verificar sempre a oximetria digital (exame nãoinvasivo), pois podem desenvolver a hipóxia silenciosa podendo evoluir para uma pneumonia grave com a falta de oxigênio na corrente sanguínea e órgãos (Sbi, 2020; Jin, 2020).

A Azitromicina, outro fármaco muito utilizado, é um antibiótico presente em muitos protocolos para COVID-19, principalmente em associação com a hidroxicloroquina que foi alvo de muitas discussões. Em relação à sua utilização na COVID-19, um dos primeiros estudos mostraram que a associação entre hidroxicloroquina e azitromicina conduzia a uma diminuição da carga viral em pacientes infectados. Porém, a OMS e OPAS (Organização Pan Americana da Saúde) chegaram ao consenso de que os pacientes avaliados estavam em estado grave quando receberam esses fármacos. Além de que não houveram diferenças na evolução do paciente que foram submetidos ao medicamento, mas sim, tiveram consequências de muitos efeitos adversos, como alterações na visão, convulsões, problemas no coração, entre outros (OMS, 2020; OPAS, 2021; Rosenberg, 2020).

Dessa forma, a OMS e a OPAS concluíram que não há dados clínicos suficientes que demonstrem benefícios ou riscos na utilização dos antibióticos em pacientes com COVID-19 (infecção viral) sem evidência de infecção bacteriana, uma vez que também induz a resistência microbiana. Assim como, a Ivermectina não é recomendada o uso para quaisquer outras finalidades diferentes daquela para os quais seu uso está devidamente autorizado (Gautret, 2020; Sbi, 2020).

Outra classe farmacológica em evidência são os corticoides, sendo sua utilização ainda controversa. A dexametasona é um corticosteroide que foi testado em pacientes hospitalizados com COVID-19 nos testes clínicos Recovery, do Reino Unido. $\mathrm{O}$ estudo mostrou que tratamento reduziu a mortalidade nos pacientes em ventilação mecânica e nos pacientes que precisavam apenas de oxigênio (OMS, 2020; OPAS, 2021). Entretanto, a OMS e o CDC (Center for Disease Control and Prevention) dos Estados Unidos, sugerem que os corticoides sejam utilizados apenas em estágios de exacerbação de asma e Doença Pulmonar Obstrutiva Crônica (DPOC) ou em casos de choque séptico (Cdc, 2020; Who, 2020).

Todos os pacientes em nenhum momento da pandemia pararam a utilização dos seus medicamentos, sendo importante nesse caso, a monitorização, pois algumas drogas possuem interações medicamentosas que podem se manifestar quando não utilizadas e monitoradas corretamente. A seguir, a Tabela 3 apresenta as interações medicamentosas significantes encontradas na terapia dos pacientes, que correspondem a $40 \%$ dos participantes. Uma das finalidades da Monitorização Terapêutica de Medicamentos (MTM) é a captação de informações sobre o nível sérico dos fármacos para avaliação e recomendação, se necessário, de ajuste de doses que permitam aderir determinados desfechos clínicos, conciliando principalmente as interações medicamentosas graves (Dasgupta, 2016; Xiao, 2020).

Tabela 3. Interações Medicamentosas encontradas entre os medicamentos utilizados pelos pacientes do ambulatório.

\begin{tabular}{cr}
\hline Medicamentos & Interação Medicamentosa \\
\hline $\begin{array}{c}\text { Azitromicina + Sinvastatina } \\
\text { Diovan HCT (80/125) + Aspirina } \\
\text { Prevent (AAS) }\end{array}$ & $\begin{array}{c}\text { A azitromicina pode aumentar os efeitos da sinvastatina. } \\
\text { A combinação desses medicamentos pode minimizar os efeitos do valsartan na redução da } \\
\text { pressal. Além disso, esses medicamentos podem afetar a funça renal, especialmente } \\
\text { quando são usados juntos com frequência ou cronicamente. }\end{array}$ \\
Atorvastatina + Lipanon & $\begin{array}{c}\text { Pode aumentar o risco de efeitos adversos da Sinvastatina, como danos ao fígado e uma } \\
\text { condição rara, mas grave, chamada rabdomiólise, que envolve a degradação do tecido muscular } \\
\text { esquelético. }\end{array}$ \\
$\begin{array}{c}\text { Prednisona + Metformina } \\
\text { Koide D + Metformina }\end{array}$ & Diminuem os efeitos da metformina, aumentando níveis de glicose \\
\hline
\end{tabular}


Dentre as interações medicamentosas encontradas na farmacoterapia dos pacientes (tabela 3) estão a azitromicina com a sinvastatina, onde a azitromicina maximiza os efeitos da sinvastatina provocando aumento dos problemas de miopatiarabdomiólise ao afetar o metabolismo da enzima hepática CYP3A4, sendo necessário uma cuidadosa monitorização. Diovan HCT com AAS provocam aumento da toxicidade de ambos podendo resultar em deterioração da função renal, particularmente em idosos ou indivíduos com depleção de volume, assim como aumentar o potássio sérico e diminuir os efeitos do valsartan por antagonismo farmacodinâmico. Do mesmo modo, por antagonismo, existe a relação da metformina com o Koide D e prednisona que favorece a diminuição dos efeitos da metformina, provocando a queda de glicose. No entanto, a interação encontrada entre a atorvastatina e o fenofibrato é caracterizada como um sinergismo farmacodinâmico (Faludi, 2017; Gerard, 2020).

O sinergismo farmacodinâmico caracteriza pelo aumento mutuamente dos efeitos de ambas as drogas, neste caso, o fenofibrato com atorvastatina poderá aumentar ainda mais o risco de rabdomiólise. Processos que envolvem a farmacocinética e farmacodinâmicas expõe os pacientes a um maior risco de efeitos adversos, sendo imprescindível a monitorização dessas drogas associadas em cada paciente. Apesar de possuir essas interferências na bibliografia, a utilização concomitante desses fármacos não ocasionou em dano imediato a saúde percebido pelos pacientes (Gerard, 2020; Malik, 2020).

No que se refere às mudanças nas condições de saúde resultantes da infecção pelo coronavírus, o parâmetro de maior prevalência na HAS e DM foi o fato de, após a pandemia o estado de saúde ter permanecido igual em $70 \%$ dos pacientes. Entre elas, a diabetes (55\%) foi a que menos se alterou com a pandemia do Covid-19.

\section{Considerações Finais}

A revisão dos dados coletados na pesquisa constatou que, para HAS e DM o questionário obteve respostas em maior prevalência que indicavam que a pandemia do COVID-19 não gerou mudanças perceptíveis na farmacoterapia dos participantes. No entanto, o vírus refletiu negativamente no estado geral de saúde dos pacientes fazendo com que muitos ficassem com sequelas, desde dores no corpo até disfunção renal e desidratação. Cerca de $70 \%$ dessas pessoas desenvolveram sequelas pelo coronavírus e $40 \%$ possuíam interações medicamentosas graves em seus tratamentos para HAS e DM isolados, bem como, concomitante com o tratamento da COVID-19, entre o Diovan HCT e AAS e entre a metformina e prednisona, respectivamente, que uma vez associados podem gerar consequências negativas como a toxicidade de ambos, tendo a necessidade de um acompanhamento farmacológico para monitorização dos mesmos.

Com o começo da pandemia e aumento do número de mortes, o mundo inteiro se mobilizou com pesquisas para entender a doença provocada pelo coronavírus e chegar ao desenvolvimento de vacinas e de um possível tratamento. Porém, a terapêutica era e continua sendo algo incerto, levando as pessoas a se automedicarem, provocando um prejuízo no seu estado clínico e terapêutico, necessitando de acompanhamento e monitorização profissional. O acompanhamento dos pacientes crônicos e principalmente idosos é essencial para o controle das doenças citadas, participantes de 40 a 70 anos representaram cerca de $60 \%$ dos pacientes que sentiram os impactos da COVID-19. Além do acompanhamento serão necessários futuros trabalhos para melhor identificar essa piora nos sintomas.

Nesse sentido, sugere-se que o acompanhamento farmacoterapêutico seja inserido em projetos de pesquisa futuros com o enfoque no atendimento clínico dos pacientes, garantindo-lhes saúde, bem-estar e qualidade de vida.

\section{Referências}

Abajo, F. J., Rodriguez-Martin, S. Lerma, V., Mejía-Abril, G., Aguilar, M., García-Luque, A., Laredo, L., Laosa, O., Centeno-Soto, G. A., Ángeles, Gálvez. M., Puerro, M., González-Rojano, E., Pedraza, L., de Pablo, I., Abad-Santos, F., Rodríguez-Mañas, L., Gil. M.; Tobías, A., Rodríguez-Miguel, A., \& Rodríguez-Puyol, (2020). D. MED-ACE2-COVID19 study group. Use of renin-angiotensin-aldosterone system inhibitors and risk of COVID-19 requiring admission to hospital: a case-population study. Lancet, 30(395) (10238), 1705-1714. 
Arnett, D. K., Blumenthal, R. S., Albert, M. A., Buroker, A. B., Goldberger, Z. D., \& Hahn, E. J. (2019). ACC/AHA Guideline on the Primary Prevention of Cardiovascular Disease. JACC, 74(10): e 177-232.

Aronson, J. K., \& Ferner, R. E. (2020). Inibidores da enzima de conversão da angiotensina (ECA) e bloqueadores do receptor da angiotensina no COVID-19 CEBM.

Bardin, L. (2011). Análise de conteúdo. Edições 70.

Barroso, W. K. S., Rodrigues, C. I. S., Bortolotto, L. A., Mota-Gomes, M. A., Brandão, A. A., \& Feitosa, ADM. (2020). Diretrizes Brasileiras de Hipertensão Arterial. Arq Bras Cardiol, PP.0-0.

Bhutani, S., \& Cooper, J. A. (2020). COVID-19 related home confinement in adults: weight gain risks and opportunities. Obesity (Silver Spring). https://doi.org/10.1002/oby.22904. https://doi.org/10.1002/oby.22904.

Bouadma, L., Lescure, F. X., Lucet, J. C., Yazdanpanah, Y., \& Timsit, J, F. (2020). Severe SARS-CoV-2 infections: practical considerations and management strategy for intensivists. Intensive Care Med.

Brasil. (2021). Ministério da Saúde. Doença pelo coronavírus. Boletim Epidemiológico Especial. Brasília.

Brasil. (2013). Ministério da Saúde. Estratégias para o cuidado da pessoa com doença crônica: hipertensão arterial sistêmica. Brasília: Ministério da Saúde, Cadernos de Atenção Básica, 37.

Brasil. (2021). Ministério da Saúde. Secretaria da Saúde. Estilo de vida saudável. Número de hipertensos cresce no mundo. Brasília: Ministério da Saúde; 2019b. http://www.saude.br/index.php/articles/123-artigos-antigos/444-numero-de hipertensos-cresce-no-mundo.

Brasil. (2017). Ministério da Saúde. Vigilância de fatores de risco e proteção para doenças crônicas por inquérito telefônico. Brasília: Ministério da Saúde, Agência Nacional de Saúde Suplementar.

Brasil. (2020). Protocolo de manejo clínico do coronavírus (COVID19) na atenção primária à saúde. Secr Atenção Primária à Saúde .

CDC. (2020). Interim Clinical Guidance for Management of Patients with Confirmed Coronavirus Disease (COVID-19).

Chaolin, H., Yeming, W. \& Xingwang, L. (2020). Clinical features of patients infected with 2019 novel coronavirus in Wuhan, China. The Lancet, 395: 497506.

Costa, C. L. A., Costa, T. M. \& Barbosa Filho, V. C. (2020). Influência do distanciamento social no nível de atividade física durante a pandemia do COVID19. Revista Brasileira de Atividade Física e Saúde, 25, e0123.

Dasgupta, A. (2016). Therapeutic Drug Monitoring of Mycophenolic Acid, 76. 10.1016/bs.acc.2016.04.001.

Daskalopoulou, S. S., Rabi, D. M., Zarnke, K. B., Dasgupta, K., Nerenberg, K., \& Cloutier, L. (2015). The 2015 Canadian Hypertension Education Program recommendations for blood pressure measurement, diagnosis, and assessment of risk, prevention, and treatment of hypertension. Can J Cardiol, 31 (5): 549-68.

Davies, M. J., D’Alessio, D. A., Fradkin, J., Kernan, W. N., Mathieu, C., \& Mingrone, G. (2020). Management of Hyperglycemia in Type 2 Diabetes. A Consensus Report by the American Diabetes Association (ADA) and the European Association the Study of Diabetes (EASD). Diabetes Care, 41(12). https://care.diabetesjournals.org/content/early/2018/09/27/dci18-0033.full-text.pdf.

Deng, L., Li, C., Zeng, Q., Liu, X., Li, X., \& Zhang, H. (2020). Arbidol combined with LPV/r versus LPV/r alone against Corona Virus Disease 2019: A retrospective cohort study. $J$ Infect.

Faludi, A. Izar, M., Saraiva, J., Chacra, A., Bianco, H., \& Afiune Neto, (2017). A. Atualização da diretriz brasileira de dislipidemias e prevenção da aterosclerose - 2017. Arquivos Brasileiros de Cardiologia [Internet], 109(1). http://www.gnresearch.org/doi/10.5935/abc.20170121

Fang, L., Karakiulakis, G., \& Roth, M. (2020). Are patients with hypertension and diabetes mellitus at increased risk for COVID-19 infection? The Lancet. Respiratory medicine. England, 116.

Ferreira, C., \& Santos, G. A. C. (2020). Métodos Laboratoriais para Diagnóstico da Infecção pelo SARS-CoV-2 [Internet], 105. Sociedade Brasileira de Patologia Clínica.

Flacco, M. E., Acuti Martellucci, C., \& Bravi, F. (2020). Tratamento com inibidores da ECA ou ARBs e risco de COVID-19 grave / letal: uma metaanálise. Heart, pmid: 32611676.

Fox, C. S., Golden, S. H., \& Anderson, C. (2017). AHA/ADA Scientific Statement: Update on prevention of cardiovascular disease in adults with type 2 diabetes mellitus in light of recent evidence. Circulation 132: 691-718.

Gabarre, P. (2020). Acute kidney injury in critically ill patients with COVID-19. Intensive Care Med, 46, 7, 1339-1348. DOI: 10.1007/s00134-020-06153-9. https://pubmed.ncbi.nlm.nih.gov/32533197/.

Gao, C., Cai, Y., Zhang, K., Zhou, L., Zhang, Y., \& Zhang, X. (2020). Association of hypertension and antihypertensive treatment with COVID-19 mortality: a retrospective observational study. Eur. heart. j. [Internet], 06];41:2058-66. https://academic.oup.com/eurheartj/article/41/22/2058/5851436.

García-Álvarez, L., Fuente-Tomás, L., Sáiz, P. A., García-Portilla, M. P., \& Bobes, J. (2020). Will changes in alcohol and tobacco use be seen during the COVID-19lockdown? 32(2):85-9. https://doi.org/10.20882/adicciones.1546» https://doi.org/10.20882/adicciones.1546.

Gautret et al. (2020). Hydroxychloroquine and azithromycin as a treatment of COVID-19: results of an open-label non-randomized clinical trial. Int $\mathbf{J}$ Antimicrob Agents. 
Gérard, A., Romani, S., Fresse, A., Viard, D., Parassol, N., \& Granvuillemin, A. (2020). "Off-label" use of hydroxychloroquine, azithromycin, lopinavirritonavir and chloroquine in COVID-19: a survey of cardiac adverse drug reactions by the French Network of Pharmacovigilance Centers. Therapie; 75:371-9.

Gil, A. C. (2008). Métodos e Técnicas de Pesquisa Social. Atlas.

Grasselli, G., Greco, M., Zanella, A., Albano, G., Antonelli, A., \& Bellani, G. (2020). Risk factors associated with mortality among patients with COVID-19 in intensive care units in Lombardy, Italy. JAMA Intern Med, 180(10):e203539.

Greenwood, B. (2014). A contribuição da vacinação para a saúde global: passado, presente e futuro. Philos Trans R Soc Lond B Biol Sei, $369: 20130433$. Guan, W., Ni, Z., \& Hu, Y. (2020). Clinical Characteristics of coronavirus disease 2019 in China. N Engl J Med. doi: 10.1056 / NEJMoa2002032.

Guo, T., Fan, Y., Chen, M., Wu, X., Zhang, L., \& He, T. (2020). Cardiovascular implications of fatal outcomes of patients with coronavirus disease 2019 (COVID-19). JAMA Cardiol.

Hollstein, T., Schulte, D., Schulz, J., Glück, A., Ziegler, A., \& Bonifacio, E. (2020). Autoantibody-negative insulin-dependent diabetes mellitus after SARSCoV-2 infection: a case report. Nature Metabolism, 2(10), 1021-1024. doi: 10.1038/s42255-020-00281-8.

Huang, C. (2020). Clinical features of patients infected with 2019 novel coronavirus in Wuhan, China. Lancet.

Hussain, A., Bhowmik, B., \& do Vale Moreira, N.C. (2020). COVID-19 and diabetes: Knowledge in progress. Diabetes Res Clin Pract, 162:108142. doi:10.1016/j.diabres.2020.108142.

Instituto de Comunicação e Informação Científica e Tecnológica em Saúde, Fundação Oswaldo Cruz. (2020). MonitoraCovid-19. https://bigdatacovid19.icict.fiocruz.br/.

International Diabetes Federation. (2015). IDF Diabetes Atlas [Internet]. 7th ed. Brussels, Belgium: International Diabetes Federation.

Jin, Y. H., Cai, L., Cheng, Z. S., Cheng, H., Deng, T., \& Fan, Y, P. (2020). A rapid advice guideline for the diagnosis and treatment of 2019 novel coronavirus (2019-nCoV) infected pneumonia (standard version). Mil Med Res, 7(1):4.

Kenneth McIntosh, M. D. (2020). Novel Coronavirus (2019-nCov). UpToDate.

Lana, R. M., Coelho, F. C., Gomes, M. F. C., Cruz, O. G., Bastos, L. S., \& Villela, D. A. M. (2020). Emergência do novo coronavírus (SARS-CoV-2) e o papel de uma vigilância nacional em saúde oportuna e efetiva. Cad Saúde Pública, 2020;36(3):1-5.

Lefèvre, F., \& Lefèvre, A. M. C. (2005). O discurso do sujeito coletivo: um novo enfoque em pesquisa qualitativa (desdobramentos). EDUSC.

Lefèvre, F., Lefèvre, A. M. C., \& Marques, M. C. C. (2009). Discurso do sujeito coletivo, complexidade e auto-organização. Ciência e saúde coletiva, Rio de Janeiro, 14, 4, 1193-1204.

Lima, C. M. O. Radiologia Brasileira. Informações sobre o novo coronavirus (COVID-19), 53.

Lopes, R. D., Macedo, A. V. S., de Barros, E., Silva, P. G. M., Moll-Bernardes, R. J., Feldman, A., \& D’Andréa Saba Arruda, G. (2020). Continuing versus suspending angiotensin-converting enzyme inhibitors and angiotensin receptorblockers: Impact on adverse outcomes in hospitalized patients withsevere acute respiratory syndrome coronavirus 2 (SARS-CoV-2)--TheBRACE CORONA Trial. Am Heart J, 226:49-59. doi: 10.1016/j.ahj.2020.05.002.

Lopez, M., Bell, K., Annaswamy. T., Juengst, S., \& Ifejika, N. (2020). COVID-19 Guia para o clínico de reabilitação: Uma revisão das manifestações e complicações não pulmonares. Am J Phys Med Rehabil, 99: 669-673.

Maddaloni, E., \& Buzzetti R. (2020). Covid-19 and diabetes mellitus: unveiling the interaction of two pandemics. Diabetes Metab Res Rev, e33213321. doi:10.1002/dmrr.3321.

Malhotra, N. (2001). Pesquisa de marketing. (3a ed.), Bookman.

Malik, M., Tahir, M, J., Jabbar, R., Ahmed, A., \& Hussain R. (2020). Self-medication during Covid-19 pandemic: challenges and opportunities. Drugs Ther Perspect, 36:565-7.

Milken Institute. (2020). COVID-19 tratamento e rastreador de vacinas, Milken Institute (2020) COVID-19 tratamento e rastreador de vacinas. https://covid19tracker.milkeninstitute.org/.

New-Onset Diabetes in Covid-19 NEJM. (2021). New England Journal Of Medicine. https://www.nejm.org/doi/full/10.1056/NEJMc2018688.

Onder, G., Rezza, G., \& Brusaferro, S. (2020). Taxa de letalidade e características de pacientes que morrem em relação ao COVID-19 na Itália. JAMA 323 (18): $1775-1776$.

OPAS - Organização Pan-Americana da Saúde. (2020). Folha informativa COVID-19 - Escritório da OPAS e da OMS no Brasil. 2021.

Organização Mundial da Saúde (OMS). (2020). Teste de diagnóstico para SARS-CoV-2. WHO/2019-nCoV/laboratory/2020.6. Genebra: OMS.

Póvoa, R., Barroso, W. S., Brandão, A. A., Jardim, P. C., Barroso, O. \& Passarelli, O. Jr. et al. (2014). I Brazilian position paper on antihypertensive drug combination. Arq Bras Cardiol, 102(3):203-10.

Précoma, D. B., Oliveira, G. M. M., Simão, A. F., Dutra, O. P., Coelho, O. R., \& Izar, M. C. O. (2019). Atualização da Diretriz de Prevenção Cardiovascular da Sociedade Brasileira de Cardiologia - 2019. Arq Bras Cardiol, 113(4):787-891.

Ramanathan, K., Antognini, D., Combes, A., Paden, M., Zakhary, B., \& Ogino, M. (2020). Planning and provision of ECMO services for severe ARDS during the COVID-19 pandemic and other outbreaks of emerging infectious diseases. Lancet Respir Med. 
Romero, D.E., \& Silva, D. R. P. (2021). Scielo. Idosos no Contexto da Pandemia da Covid-19 no Brasil: Efeitos nas Condições de Saúde, Renda e Trabalho. Cad. Saúde Pública 37. https://scielosp.org/article/csp/2021.v37n3/e00216620/.

Roncon, L., Zuin, M., Rigatelli, G., \& Zuliani, G. (2020). Pacientes diabéticos com infecção por COVID-19 apresentam maior risco de admissão na UTI e desfecho desfavorável em curto prazo.

Rosenberg et al. (2020). Association of treatment with hydroxychloroquine or azithromycin with in-hospital mortality in patients with COVID-19 in New York State. JAMA.

Rubino, F., Amiel, S. A., Zimmet, P., Alberti, G., Bornstein, S., \& Eckel, R. H. (2020). New-Onset Diabetes in Covid-19. N Engl J Med, 383(8):789-790. doi:10.1056/nejmc2018688.

SBI - Sociedade Brasileira de Imunologia. (2020). Parecer Científico da Sociedade Brasileira de Imunologia sobre a utilização da Cloroquina/Hidroxicloroquina para o tratamento da COVID-19.

Skyler, J. S., Bakris, G. L., Bonifacio, E., Darsow, T., Eckel, R. H., \& Groop, L. (2017). Differentiation of diabetes by pathophysiology, natural history, and prognosis. Diabetes, 66(2):241-55.

Sociedade brasileira de infectologia atualizações e recomendações sobre a COVID-19. (2020). https://infectologia.org.br/wpcontent/uploads/2020/12/atualizacoes-e-recomendacoes-covid-19.pdf.

Sociedade Brasileira de Patologia Clínica e Medicina Laboratorial - SBPC/ML. (2020). Métodos laboratoriais para diagnóstico da infecção pelo SARS-CoV-2. Recomendações da Sociedade Brasileira de Patologia Clínica/Medicina Laboratorial.

Solon Júnior, L. J. F., Fortes, L. S., \& Barbosa, B. T. et al. (2020). Home-based exercise during confinement in COVID-19 pandemic and mental health in adults: a cross-sectional comparative study. Revista Brasileira de Atividade Física e Saúde, 25, e0120.

Task Force of the Latin American Society of Hypertension. (2017). Guidelines on the management of arterial hypertension and related comorbidities in Latin America. Journal of Hypertension, 35(8):1529-45.

Vaduganathan, M., Vardeny, O., Michel, T., McMurray, J. J., Pfeffer, M. A., \& Solomon, S. D. (2020). Renin-angiotensin-aldosterone system inhibitors in patients with Covid-19. N Engl J Med, 382:1653-59. doi: 10.1056/NEJMsr2005760.

Van Zyl-Smit, R. N., Richards, G., \& Leone, F. T. (2020). Tobacco smoking and COVID-19 infection. Lancet Respir Med,. 8(7): 664-5. https://doi.org/10.1016/S2213-2600(20)30239-3.

Wald, D. S., Law, M., Morris, J. K., Bestwick, J. P., \& Wald, N. J. (2014). Combination therapy versus monotherapy in reducing blood pressure: metaanalysis on 11,000 participants from 42 trials. $J$ Med, 122(3):290-300.

Wang, D., Hu, B., Hu, C., Zhu, F., Liu, X., \& Zhang J. (2020). Clinical Characteristics of 138 Hospitalized Patients With 2019 Novel Coronavirus-Infected Pneumonia in Wuhan, China. JAMA.

Wang, W., Xu, Y., Gao, R., Lu, R., Han, K., \& Wu, G. (2020). Detection of SARS-CoV-2 in Different Types of Clinical Specimens. JAMA, 323(18):1843-1844. WHO. (2020). Clinical management of severe acute respiratory infection (SARI) when COVID-19 disease is suspected.

WHO. (2020). Coronavirus disease 2019 (COVID-19) Situation Report- 51. Geneva. https://www.who.int/docs/default-source/coronaviruse/situationreports/20200311-sitrep-51-covid-19.pdf?sfvrsn=1ba62e57_10.

WHO. (2021). Draft landscape of COVID-19 candidate vaccines. World Health Organization. https://www. who.int/docs/default-source/coronaviruse/novelcoronavirus-landscape-covid-.

Williams, B., Mancia, G., Spiering, W., Agabiti, R. E., Azizi, M., \& Burnier, M. (2018). ESC/ESH Guidelines for the management of arterial hypertension: The Task Force for the management of arterial hypertension of the European Society of Cardiology and the European Society of Hypertension: The Task Force for the management of arterial hypertension of the European Society of Cardiology and the European Society of Hypertension. $J$ Hypertens. 36(10):1953-2041.

World Health Organization (WHO). (2020). Laboratory testing for coronavirus disease 2019 (COVID-19) in suspected human cases. Interim guidance. Reference: WHO/COVID-19/laboratory/2020.5.

World Health Organization. (2020). Clinical management of severe acute respiratory infection when novel coronavirus (nCoV) infection is suspected.

World Health Organization. (2016). Global report on diabetes. Geneva.

World Health Organization. (2020). Statement on the second meeting of the international health regulations (2005) emergency committee regarding the outbreak of novel coronavirus (2019-nCoV). Geneva: World Health Organization. https://www.who.int/news-room/ detail/30-01-2020-statement-on-thesecond-meetingof-the-international-health-regulations-(2005) emergency-committee-regarding-the-outbreak-ofnovel-coronavirus-(2019-ncov).

Xiao, S. Y., Wu, Y., \& Liu, H. (2020). Evolving status of the 2019 novel coronavirus infection: Proposal of conventional serologic assays for disease diagnosis and infection monitoring. J Med Virol, 92(5):464-7.

Zheng, Z., Peng, F., Xu, B., Zhao, J., Liu, H., \& Peng, J. (2020). Fatores de risco de casos críticos e mortais de COVID-19: uma revisão sistemática da literatura e meta-análise. J Infect.

Zhou, F., Yu, T., Du, R., Fan, G., Liu, Y., \& Liu, Z. et al. (2020). Clinical course and risk factors for mortality of adult inpatients with COVID-19 in Wuhan, China: a retrospective cohort study. Lancet, 395(10229):1054-62.

Zhu, J., Ji, P., Pang, J., Zhong, Z., Li, H., \& He, C. (2020). Características clínicas de 3.062 pacientes com COVID-19: uma meta-análise. J Med Virol. 